\title{
A Two-Dimensional DOA Estimation Method Based on Virtual Extension of Sparse Array
}

\author{
Guibao Wang $\mathbb{D},{ }^{1}$ Peiyao Zhao $\mathbb{D},{ }^{2}$ Lanmei Wang $\mathbb{D},{ }^{3}$ Xiangyu Wang $\mathbb{D},{ }^{3}$ Huan Wang $\mathbb{D}{ }^{3}$ \\ and Zhongpeng Zhang $\mathbb{D}^{1}$
}

\author{
${ }^{1}$ School of Physics and Telecommunication Engineering, Shaanxi University of Technology, Hanzhong 723001, China \\ ${ }^{2}$ School of Telecommunications Engineering, Xidian University, Xi'an 710071, China \\ ${ }^{3}$ School of Physics and Optoelectronic Engineering, Xidian University, Xi'an 710071, China
}

Correspondence should be addressed to Lanmei Wang; lmwang@mail.xidian.edu.cn

Received 3 May 2020; Revised 21 April 2021; Accepted 14 May 2021; Published 25 May 2021

Academic Editor: Antonis G. Dimitriou

Copyright ( 92021 Guibao Wang et al. This is an open access article distributed under the Creative Commons Attribution License, which permits unrestricted use, distribution, and reproduction in any medium, provided the original work is properly cited.

The interelement spacing of a coprime array breaks through the half-wavelength limitation, so that a larger array aperture can be obtained with a fixed number of array elements. In this paper, the symmetry of the noncircular signal is used to virtually expand the L-shaped array into an orthogonal cross array. Furthermore, the virtual received signal of the augmented array is obtained by the second-order statistic of the received data. Decoupling and dimension reduction of elevation and azimuth are realized by a $z$ axis subarray and $y$-axis subarray. Finally, the sparse reconstruction of the signal is realized by the minimum absolute convergence and selection operator method. This method can enlarge the aperture and freedom of array and has higher accuracy and resolution of DOA estimation. It has the advantages of automatic parameter pairing without additional pairing operation and is effective for coherent and incoherent signals. The final numerical simulation results prove the effectiveness of the method in this paper.

\section{Introduction}

Direction of arrival (DOA) estimation is an important research content of array signal processing, which is widely used in navigation, communication, and radar $[1,2]$. After decades of development, a complete set of processing theory and method for the estimation of DOA has been formed. Subspace-like high-resolution algorithms such as MUSIC and ESPRIT break through the restriction of Rayleigh limits and have good highresolution performance $[3,4]$. In the actual environment, due to the existence of multipath effect and electromagnetic interference, the signals received by the array are often coherent signals. The existence of coherent signals leads to the performance degradation or even complete ineffectiveness of the traditional subspace-like high-resolution algorithms. The existence of the coherent signal results in the rank loss of the data covariance matrix. The rank of the data covariance matrix must be restored before the subspace algorithm can be applied to the DOA estimation of the coherent signal, which also leads to the increase of the calculation amount.

The application of a coprime array makes the degree of freedom of DOA estimation no longer limited by the number of physical array elements, thus greatly reducing the hardware overhead $[5,6]$. The interelement spacing of the coprime array based on the sparse model is larger than half a wavelength, so the aperture of the array is effectively expanded and the resolution is improved. In recent years, the coprime array has become a research hotspot in the field of array signal processing. In $[7,8]$, a virtual array model based coprime array is presented [9]. In [10], a spatially smooth DOA estimation algorithm based on the continuous virtual array element is presented. However, this method leads to the abandonment of partial array elements due to the need of continuous virtual arrays. In order to compensate for this deficiency, a DOA algorithm based on the covariance matrix reconstruction of the coprime array is proposed in [11]. In [12], a two-dimensional angle estimation based on the L-shaped coprime array is proposed. In [13], the azimuth of 
the signals' source is estimated by constructing the cumulant matrix, which reduces the aperture loss of the array. In [14], a two-dimensional direction-finding algorithm for sparsely $\mathrm{L}$-shaped coprime arrays is represented.

Because of its unique statistical characteristics, the noncircular signals can be processed by using both the covariance matrix and the elliptic covariance matrix of the received data at the receiving end, which can effectively improve the performance of signal detection and estimation. With the wide application of noncircular signals in communication and other fields, such as common BPSK and AM signals, noncircular signal direction finding has become a new hot spot of research $[15,16]$.

The compressed sensing technology can achieve highefficiency signal processing under the condition of undersampling by compressing redundant information. The compressed sensing algorithm does not require the eigendecomposition of the data correlation matrix, so it can directly distinguish the coherent signals without additional decoherence processing. The degree of freedom of the nonuniform array can be fully utilized, unlike the spatial smoothing method and the Toeplitz method, which utilize only half of the degrees of freedom $[17,18]$.

In this paper, the advantages of the sparse coprime array and the characteristics of the noncircular signal are combined to receive the data from the actual array to obtain the received data of the symmetric virtual array, and the actual and virtual array data constitute the whole array data, so as to realize the further expansion of the array data. By vectorizing the covariance matrix of the whole array data and by deduplication, a new array receiving data is obtained. Combined with the structural advantages of the L-shaped array, the two-dimensional DOA estimation of the incoherent noncircular signal is realized by the compressed sensing algorithm, which enlarges the aperture of the array and the degree of freedom of the array to a certain extent and improves the resolution and estimation accuracy of the DOA.

\section{Array Signal Model}

2.1. Array Architecture. As shown in Figure 1, the coprime array is a kind of nonuniform array, which consists of two subarrays.

Here, the number of elements of the two subarrays satisfies the relatively prime relationship, and the interelement spacing also satisfies the relatively prime relationship, and the interelement spacing is no longer limited to half wavelength, so it is a sparse array. The structure of the coprime array and its subarrays is shown in Figure 2.

By contrast, as shown in Figures 3 and 4, the array element position of the symmetric uniform array and symmetric coprime array is $(0, d, 2 d, 3 d, \ldots,(M-1) d)$ and $(0, \mathrm{Md}, \mathrm{Nd}, 2 \mathrm{Md}, 2 \mathrm{Nd}, \ldots,(M-1) \mathrm{Nd},(N-1) \mathrm{Md})$.

The received data of the negative half-axis are also obtained by virtual expansion based on the symmetry of the circular signal, and virtual expansion is carried out by vectorization of the data correlation matrix. The array element position of the negative half-axis of the uniform array and coprime

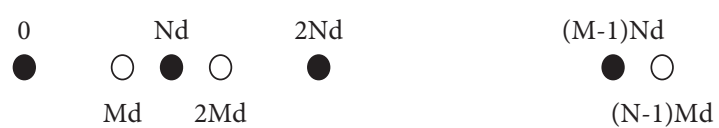

FIgURE 1: The structure of the coprime array.

array is $(-d,-2 d,-3 d, \ldots,-(M-1) d)$ and $(-d,-\mathrm{Nd},-2 \mathrm{Md},-2 \mathrm{Nd}, \ldots,-(M-1) \mathrm{Nd},-(N-1) \mathrm{Md})$.

2.2. Receiving Data Model. The receiving array is an L-shaped array composed of a coprime subarray $Z_{a}$ distributed in the $z$-axis and a coprime subarray $Y_{a}$ distributed in the $y$-axis. The coprime array is composed of two sparse uniform arrays with the first element overlapped; one array is $N$ array elements with interelement spacing $\mathrm{Md}$, and the other array is $M$ array elements with interelement spacing Nd. $M$ and $N$ are two mutually prime numbers. $d$ is a fixed length, which is less than or equal to the minimum half wavelength. The schematic diagram of the array structure is shown in Figure 5.

The array element numbers of the subarray $Z_{a}$ and $Y_{a}$ are both $Q=N+M-1$, and the corresponding array element coordinates are, respectively, $\widetilde{z}=\left[\widetilde{z}_{1}, \widetilde{z}_{2}, \ldots, \widetilde{z}_{q}, \ldots, \widetilde{z}_{Q}\right] d$ and $\tilde{y}=\left[\widetilde{y}_{1}, \widetilde{y}_{2}, \ldots, \widetilde{y}_{q}, \ldots, \widetilde{y}_{Q}\right] d$, where $\widetilde{z}_{q}$ and $\widetilde{y}_{q}$ are integers, $1 \leq q \leq Q$.

$\mathrm{K}$ far-field, coherent, narrowband noncircular signals $\left(\theta_{k}, \phi_{k}\right)$ are incident on the receiving array from different directions. $\theta_{k} \in[0, \pi]$ is the elevation angle of the $k$ th signal, that is, the angle between the incident signal and the positive direction of the $z$-axis, and $\phi_{k} \in[-\pi / 2, \pi / 2]$ is the azimuth angle of the $k$ th incident signal, that is, the angle between the projection of the incident signal on the xoy plane and the positive direction of the $x$-axis. The received signal of the $z$ axis subarray $Z_{a}$ is

$$
Z_{1}(t)=\left[z_{1}(t), \ldots, z_{q}(t), \ldots, z_{Q}(t)\right]^{T}=A_{z} S(t)+N_{z}(t),
$$

where $z_{q}(t)=\sum_{k=1}^{K} s_{k}(t) e^{j\left(\mu_{k} \tilde{z}_{q}\right)}+n_{z q}(t)$ is the received signal of the $q$-th array element on the $z$-axis, $S(t)=\left[s_{1}(t), \ldots, s_{k}(t), \ldots, s_{K}(t)\right]^{T}$ is the incident signal matrix, $N_{z}(t)$ is the noise of the $z$-axis subarray, and $A_{z}=$ $\left[a_{z}\left(\theta_{1}\right), \ldots, a_{z}\left(\theta_{k}\right), \ldots, a_{z}\left(\theta_{K}\right)\right]$ is the array steering vector of the $\sim \sim^{z \text {-axis }} \sim$ subarray. $a_{z}\left(\theta_{k}\right)=\left[e^{j \mu_{k} \tilde{z}_{1}}, e^{j \mu_{k}} \tilde{z}_{2}, \ldots, e^{j \mu_{k} \tilde{z}_{q}}, \ldots, e^{j \mu_{k} \tilde{z}_{Q}}\right]^{T}$ is the array steering vector corresponding to the $k$ th signal, $n_{z q}(t)$ is the real array noise received by the $q$-th array element on the $z$ axis subarray, $\mu_{k}=-2 \pi d \cos \theta_{k} / \lambda, \lambda$ is the wavelength of the incident signal, and $s_{k}(t)$ is the $k$ th incident signal, $1 \leq k \leq K$.

The received signal of the $y$-axis subarray $Y_{a}$ is

$$
Y_{1}(t)=\left[y_{1}(t), \ldots, y_{q}(t), \ldots, y_{Q}(t)\right]^{T}=A_{y} S(t)+N_{y}(t),
$$

where $y_{q}(t)=\sum_{k=1}^{K} s_{k}(t) e^{j\left(\varphi_{k} \tilde{y}_{q}\right)}+n_{y q}(t)$ is the received signal of the $q$-th array element on the $y$ axis, $A_{y}=\left[a_{y}\left(\theta_{1}, \phi_{1}\right), \ldots, a_{y}\left(\theta_{k}, \phi_{k}\right), \ldots, a_{y}\left(\theta_{K}, \phi_{K}\right)\right]$ is the array steering vector of the $y$-axis subarray, and $N_{y}(t)$ is the 


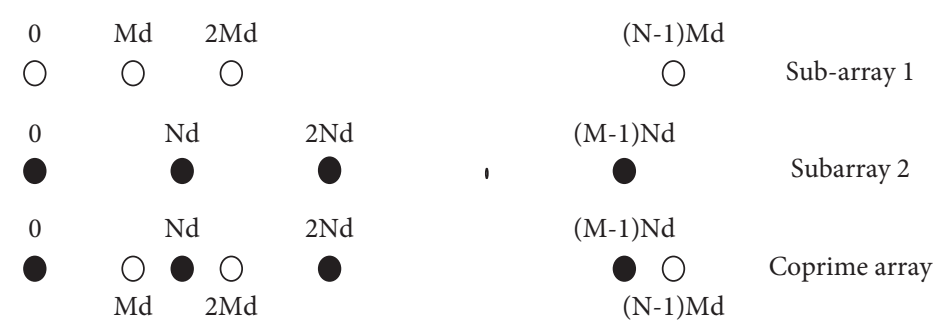

FIGURE 2: The structure of the coprime array and two subarrays.

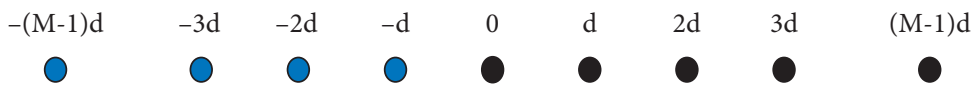

FIGURE 3: The structure of the symmetric uniform array, where the black array elements are real elements and the blue array elements are the corresponding virtual array elements.

$\begin{array}{ccccccccc}(1-\mathrm{M}) \mathrm{Nd} & -2 \mathrm{Nd} & -\mathrm{Nd} & 0 & \mathrm{Nd} & 2 \mathrm{Nd} & (\mathrm{M}-1) \mathrm{Nd} \\ 0 & \bigcirc & 0 & \bigcirc & 0 & \bigcirc & \bigcirc & \bigcirc & \bigcirc \\ (1-\mathrm{N}) \mathrm{Md} & & -2 \mathrm{Md} & -\mathrm{Md} & & \mathrm{Md} & 2 \mathrm{Md} & & (\mathrm{N}-1) \mathrm{Md}\end{array}$

Figure 4: The structure of the symmetric coprime array, where the white and black array elements are the real elements of the two subarrays and the blue and purple array elements are the virtual array elements corresponding to the real array elements.

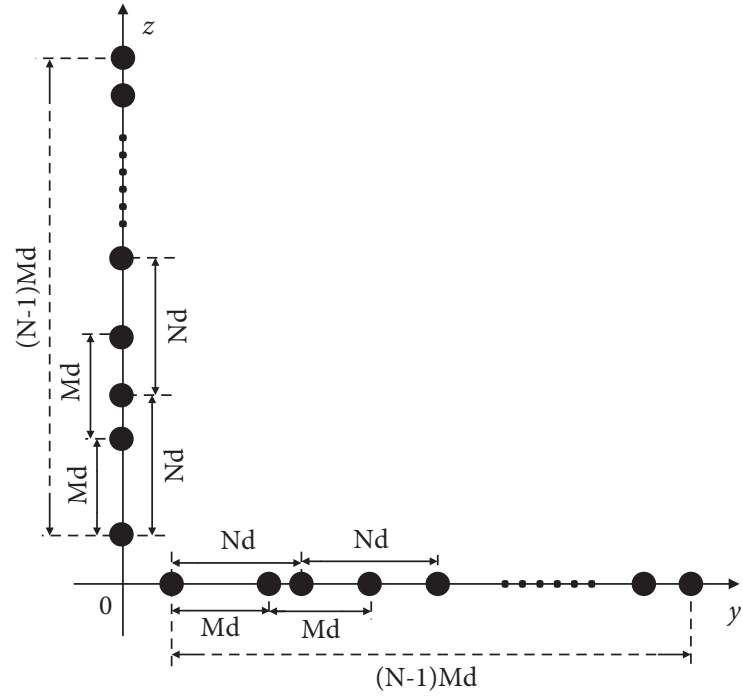

Real array element

FIgURE 5: The schematic diagram of the L-shaped array structure.

real array noise of the $y$-axis subarray. $a_{y}\left(\theta_{k}, \phi_{k}\right)=\left[e^{j \varphi_{k} \widetilde{y}_{1}}, e^{j \varphi_{k} \widetilde{y}_{2}}, \ldots, e^{j \varphi_{k} \widetilde{y}_{q}}, \ldots, e^{j \varphi_{k} \widetilde{y}_{Q}}\right]^{T}$ is the $y$ axis subarray steering vector corresponding to the $k$ th signal, $n_{y q}(t)$ is noise received by the $q$-th array element on the $y$ axis subarray, and $\varphi_{k}=-2 \pi d \sin \theta_{k} \sin \phi_{k} / \lambda$.
2.3. Virtual Expansion Array. The array elements of the original array are arranged in the positive half-axis of the coordinate axis. Using the characteristics of the noncircular signal, the received data of the negative half-axis is obtained by taking the conjugate operation to the received data of the positive half-axis array element.

In Figure 6, the array elements of the virtual subarrays $Z_{a}^{\prime}$ and $Y_{a}^{\prime}$ are represented by hollow circles, and those of the actual subarrays $Z_{a}$ and $Y_{a}$ are represented by solid circles.

The noncircular signal is actually the real signal, which satisfies the following relationship:

$$
S(t)=S^{*}(t),
$$

where $(\cdot)^{*}$ denotes the conjugate operation.

The received signal $Z_{1}(t)$ of the $z$-axis subarray $Z_{a}$ and the received signal $Y_{1}(t)$ of the $y$-axis subarray $Y_{a}$ are, respectively, conjugated to obtain the received data of the virtual array:

$$
\begin{aligned}
& Z_{2}(t)=\left[z_{\mathrm{Q}}^{*}(t), z_{\mathrm{Q}-1}^{*}(t), \ldots, z_{q}^{*}(t), \ldots, z_{1}^{*}(t)\right]^{T}, \\
& Y_{2}(t)=\left[y_{\mathrm{Q}}^{*}(t), y_{\mathrm{Q}-1}^{*}(t), \ldots, y_{q}^{*}(t), \ldots, y_{1}^{*}(t)\right]^{T},
\end{aligned}
$$

where $(\cdot)^{T}$ denotes the transpose of the matrix, $z_{q}^{*}(t)=z_{-q}(t)$, and $y_{q}^{*}(t)=y_{-q}(t)$.

The real and virtual array received signal construct the whole array received data:

$$
\begin{aligned}
& Z(t)=\left[\begin{array}{l}
Z_{2}(t) \\
Z_{1}(t)
\end{array}\right]=\left[z_{-Q}(t), \ldots, z_{-1}(t), z_{1}(t), \ldots, z_{Q}\right]^{T}=\widetilde{A}_{z} S(t)+\widetilde{N}_{z}(t), \\
& Y(t)=\left[\begin{array}{l}
Y_{2}(t) \\
Y_{1}(t)
\end{array}\right]\left[y_{-Q}(t), \ldots, y_{-1}(t), y_{1}(t), \ldots, y_{Q}\right]^{T}=\widetilde{A}_{y} S(t)+\tilde{N}_{y}(t) .
\end{aligned}
$$




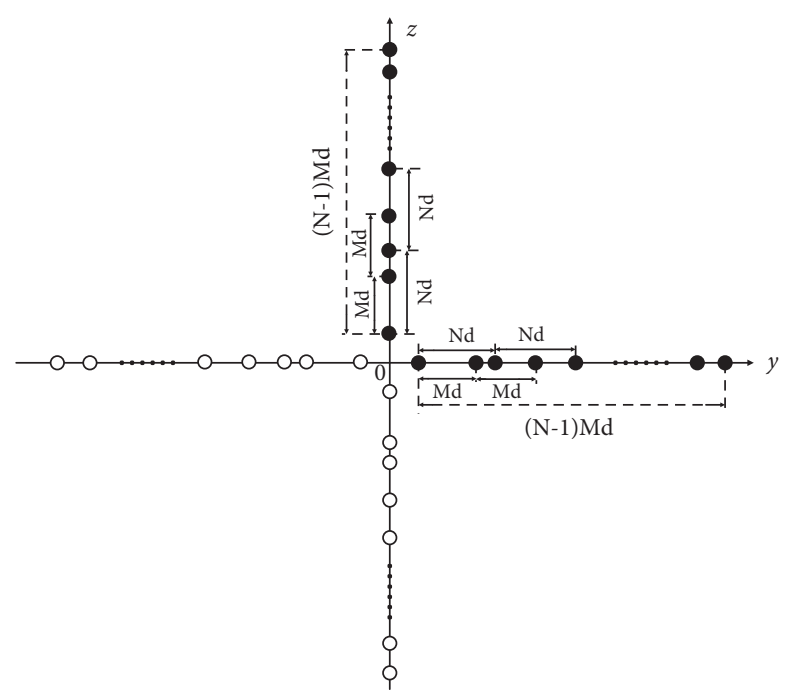

- Real array element

o Virtual array element

FIGURE 6: The schematic diagram after virtual expansion.

The steering vector in $z$-axis direction $\widetilde{A}_{z}=\left[\widetilde{a}_{z}\left(\theta_{1}\right), \ldots, \widetilde{a}_{z}\left(\theta_{k}\right), \ldots, \widetilde{a}_{z}\left(\theta_{K}\right)\right]$ is only related to the elevation angle, the steering vector in the $y$-axis direction $\widetilde{A}_{y}=\left[\tilde{a}_{y}\left(\theta_{1}, \phi_{1}\right), \ldots, \tilde{a}_{y}\left(\theta_{k}, \phi_{k}\right), \ldots, \tilde{a}_{y}\left(\theta_{K}, \phi_{K}\right)\right]$ is related to the elevation and azimuth angles, $\tilde{N}_{z}(t)$ and $\tilde{N}_{y}(t)$ are, respectively, and the whole array noises of $y$-axis and $z$-axis subarray, where $\widetilde{a}_{z}\left(\theta_{k}\right)=\left[e^{-j \mu_{k} z_{Q}} \ldots, e^{-j \mu_{k} z_{q}}, \ldots, e_{\sim}^{-j \mu_{k} \widetilde{z}_{1}}\right.$, $\left.e^{j \mu_{k} \widetilde{z}_{1}} \ldots, e^{j \mu_{k} \widetilde{z}_{q}}, \ldots, e^{j \mu_{k} z_{Q}}\right]^{T}$ and $\widetilde{\widetilde{a}}_{y}\left(\theta_{k}, \phi_{k}\right)=\left[e^{-j \varphi_{k} \widetilde{y}_{Q}} \ldots\right.$, $e^{-j \varphi_{k} \hat{y}_{q}}, \ldots, \quad e^{j \varphi_{k} y_{2}}, e^{-j \varphi_{k} \tilde{y}_{1}}, e^{j \varphi_{k} \tilde{y}_{1}}, e^{j \varphi_{k} \tilde{y}_{2}}, \ldots, e^{j \varphi_{k} \ddot{y}_{q}}$, $\left.\ldots, e^{j \varphi_{k} \tilde{y}_{Q}}\right]^{T}$; then, the position coordinate of the array elements of the whole array is as follows:

$$
\begin{aligned}
\tilde{z}_{a}=\tilde{y}_{a}= & {[-N M,-(M-1) N, \ldots,-N,-M, M, N, \ldots} \\
& \cdot(M-1) N, N M] d,
\end{aligned}
$$

where $\mathrm{M}$ and $\mathrm{N}$ are two coprime numbers, $M<N$, and $d$ is less than or equal to the half wavelength of the incident signal.

\section{Virtual Extended DOA Estimate Based on Vectorizaiton of the Covariance Matrix}

The covariance matrix of data received by the whole array is

$$
\begin{aligned}
& R_{z z}=E\left[Z Z^{H}\right]=\widetilde{A}_{z} R_{s} \widetilde{A}_{z}^{H}+\sigma_{n z}^{2} I_{2 Q}, \\
& R_{y y}=E\left[Y Y^{H}\right]=\widetilde{A}_{y} R_{s} \widetilde{A}_{y}^{H}+\sigma_{n y}^{2} I_{2 Q},
\end{aligned}
$$

where $\sigma_{n z}^{2}$ and $\sigma_{n y}^{2}$ denote the white noise power of $z$-axis and $y$-axis subarrays, respectively, $I_{2 Q}$ represents the identity matrix of $2 Q \times 2 Q, R_{s}=E\left[S(t) S^{H}(t)\right]$ is the signal correlation matrix, and $(\cdot)^{H}$ denotes the complex conjugate transpose.

The data covariance matrix $Z_{v}$ and $Y_{v}$ are vectorized to obtain the equivalent received data of the array under the augmented array, that is,

$$
\begin{aligned}
& Z_{v}=\operatorname{vec}\left(R_{z z}\right)=\left(\widetilde{A}_{z}^{*} \odot \widetilde{A}_{z}\right) P+\sigma_{n}^{2} I_{4 Q^{2}}=B_{z} P+\sigma_{n z}^{2} I_{4 Q^{2}}, \\
& Y_{v}=\operatorname{vec}\left(R_{y y}\right)=\left(\widetilde{A}_{y}^{*} \odot \widetilde{A}_{y}\right) P+\sigma_{n}^{2} I_{4 Q^{2}}=B_{y} P+\sigma_{n y}^{2} I_{4 Q^{2}},
\end{aligned}
$$

where $Z_{v}$ and $Y_{v}$ are the column vectors of $4 Q^{2} \times 1$, the rank of the two matrix is $1, \operatorname{vec}(\cdot)$ denotes the vectorization of the matrix, $\odot$ represents the Khatri-Rao product, $I_{4 Q^{2}}$ represents the identity column vector of $4 Q^{2} \times 1$, and $P=\operatorname{vec}\left(R_{s}\right)$.

$$
\begin{gathered}
B_{z}=\widetilde{A}_{z}^{*} \odot \widetilde{A}_{z}=\left[a_{z}^{*}\left(\theta_{1}\right) \otimes a_{z}\left(\theta_{1}\right), \cdots, a_{z}^{*}\left(\theta_{K}\right) \otimes a_{z}\left(\theta_{K}\right)\right], \\
B_{y}=\widetilde{A}_{y}^{*} \odot \widetilde{A}_{y}=\left[a_{y}^{*}\left(\theta_{1}, \phi_{1}\right) \otimes a_{y}\left(\theta_{1}, \phi_{1}\right), \ldots, a_{y}^{*}\left(\theta_{K}, \phi_{K}\right) \otimes a_{y}\left(\theta_{K}, \phi_{K}\right)\right],
\end{gathered}
$$

where $\otimes$ represents the Kronecker product. $B_{z}$ and $B_{y}$ are often not the steering vectors of the uniform array. Using the method of transforming the coprime array into the uniform array, $Z_{V}$ and $Y_{V}$ are transformed into the data $\widetilde{Z}_{V}$ and $\widetilde{Y}_{V}$ of the uniform augmented array, and the steering vectors $B_{z}$ and $B_{y}$ are transformed into the steering vectors $\widetilde{B}_{z}$ and $\widetilde{B}_{y}^{z}$ of the uniform augmented array. If the MUSIC method or ESPRIT method is used, the rank of the matrix needs to be restored by means of spatial smoothing and other methods, and the array will have half of the degree-offreedom loss. Considering that the compressed sensing method does not require the decomposition of the data covariance matrix and is not affected by rank loss, the compressed sensing method is used to estimate the DOA of the signal.

3.1. Estimation of Elevation Angle. The estimation of elevation angle is obtained by using the data received from coprime subarrays arranged along the $z$-axis. From equation (9), it can be seen that the steering vector of the $z$-axis subarray is a single-valued function of the elevation angle. 
The overcomplete elevation angle sparse dictionary $\widetilde{B}_{z}(\bar{\theta}) \in C^{F \times N_{\theta}}$ is constructed according to the position of the array elements of the augmented array, where $\bar{\theta}$ is the potential signal of elevation angle and $F$ is the number of elements of the uniform augmented array. The equivalent received data $\widetilde{Z}_{v}$ under the $z$-axis augmented array can be expressed as

$$
\widetilde{Z}_{v}=\widetilde{B}_{z}(\bar{\theta}) x^{\prime}+\sigma_{n z}^{2} I_{F},
$$

where $x^{\prime} \in C^{N_{\theta} \times 1}$ represents the variance of the potential signals, $x^{\prime}$ has a sparse structure and $\mathrm{K}$ nonzero lines, each nonzero position corresponds to the DOA of the sound source signal, $N_{\theta}$ is the number of the potential elevation angle signal, $N_{\theta} \gg K, N_{\theta} \gg F$, and $I_{F}$ represents the identity matrix of $F \times F$.

The 1 norm $\hat{x}=\arg \min \left\|x^{\prime}\right\|_{1}$ satisfying the constraint $\left\|\widetilde{Z}_{v}-\widetilde{B}_{z}(\bar{\theta}) x^{\prime}-\sigma_{n z}^{2} x_{F}^{\prime}\right\|_{2} \leq \varepsilon_{1}$ obtains $x^{\prime} . x^{\prime}$ is a column vector, each of its elements corresponds to a column in dictionary $\widetilde{B}_{z}(\bar{\theta})$, a column in dictionary corresponds to an incidence angle, and $x^{\prime}$ has $\mathrm{K}$ nonzero elements, which correspond to $\mathrm{K}$ incidence angles. With the consideration of noise, the elevation angle $\widehat{\theta}_{k}$ can be solved by the minimum absolute convergence method and selection operator method, and the optimization problem of formula (12) can be solved by the OMP optimization package in MATLAB.

$$
\widehat{x}=\underset{x^{\prime}}{\arg \min }\left\|x^{\prime}\right\|_{1} \quad \text { s.t. }\left\|\widetilde{Z}_{v}-\widetilde{B}_{z}(\bar{\theta}) x^{\prime}-\sigma_{n z}^{2} I_{F}\right\|_{2} \leq \varepsilon_{1},
$$

where $\|\cdot\|_{1}$ and $\|\cdot\|_{2}$ denote the 1-norm and 2-norm of matrices and $\varepsilon_{1}$ is the noise constraint, and cross validation is used to select the appropriate value.

3.2. Estimation of Azimuth Angle. The estimates of azimuth angle can be achieved by using the data received from coprime subarrays arranged along the $y$-axis. It can be seen from formula (10) that the steering vector of the $y$-axis subarray is a function of elevation and azimuth angle. Substituting the estimated value of the elevation angle, the overcomplete azimuth angle sparse dictionary $\widetilde{B}_{y}(\bar{\phi}) \in C^{F \times N_{\phi}}$ is constructed according to the array element position of the $y$-axis augmented array, where $\bar{\phi}$ is the potential signal of azimuth angle. The equivalent received data $\widetilde{Y}_{v}$ under the $z$ axis augmented array can be represented as

$$
\widetilde{Y}_{v}=\widetilde{B}_{y}(\bar{\phi}) y^{\prime}+\sigma_{n y}^{2} I_{F},
$$

where $y^{\prime} \in C^{N_{\phi} \times 1}$ indicates the variance of the potential signals, $y^{\prime}$ has a sparse structure and $\mathrm{K}$ nonzero lines, and each nonzero position corresponds to the DOA of the sound signal. $N_{\phi}$ is the number of potential azimuth angle, $N_{\phi} \gg K, N_{\phi} \gg F$.

In the case of considering noise, the azimuth angle can be estimated by solving the $\ell_{1}$ norm of equation (14); the optimization procedure of formula (14) is similar to that of formula (12):

$$
\hat{y}=\underset{y^{\prime}}{\arg \min }\left\|y^{\prime}\right\|_{1} \quad \text { s.t. }\left\|\widetilde{Y}_{v}-\widetilde{B}_{y}(\bar{\phi}) y^{\prime}-\sigma_{n y}^{2} I_{F}\right\|_{2} \leq \varepsilon_{2},
$$

where $\varepsilon_{2}$ is the noise constraint, and cross validation is used to select the appropriate value.

The parameter estimation algorithm of compressed sensing dimension reduction based on the L-shaped array is proposed. Thanks to the proposed $\widetilde{B}_{z}(\bar{\theta})$ in $(12)$ and $\widetilde{B}_{y}(\bar{\phi})$ in (14) being one-dimensional dictionaries, we achieve decoupling of the elevation and azimuth. The automatic matching of azimuth and elevation angles does not need an additional pairing operation, which reduces the amount of calculation. If MUSIC or ESPRIT algorithm is used because $\widetilde{Z}_{v}$ in (11) and $\widetilde{Y}_{v}$ in (13) are column vectors with rank 1 , it is necessary to reconstruct the data matrix with the idea of spatial smoothing to restore the rank of the matrix, resulting in half loss of the array aperture. In this paper, the optimization equations of formulas (12) and (14) are used to solve the problem by using compressed sensing, which does not need signal subspace, does not need to restore the rank of the matrix, and does not have the loss of the array aperture. The compressed sensing algorithm can process coherent and incoherent signals.

\section{Simulation and Performance Analysis}

The receiving array is an L-shaped array composed of a coprime subarray $Z_{a}$ distributed in the $z$-axis and a coprime subarray $Y_{a}$ distributed in the $y$-axis. The parameters of two coprime arrays take $M=2, N=3$, and the positions of the array elements are $(0,2 \mathrm{~d}, 3 \mathrm{~d}, 4 \mathrm{~d})$, where $d=\lambda / 2, \lambda$ is the incident signal wavelength. Considering that two far-field narrowband signals are incident on the array, the signals are independent of each other. The incident angles of the two signals are, respectively, $\left(25^{\circ}, 20^{\circ}\right)$ and $\left(70^{\circ}, 50^{\circ}\right)$, and the noise of the array is Gaussian white noise. The number of Monte Carlo experiments corresponding to each SNR is 50 times. For the sake of convenience, the array structure used in this paper is called the symmetric coprime array, and the corresponding DOA estimation method is called the proposed method.

Simulation 1. The elevation and azimuth angles estimated by the proposed method are simulated. The SNR is $10 \mathrm{~dB}$. The number of snapshots is 200 , that is to say, all array sensors are sampled 200 times synchronously, and 200 synchronous sampling data are, herein, obtained. The more the snapshots, the more the information accumulated and the more accurate the estimation results. Especially for the subspace method, the number of snapshots is too small, the performance is degraded, and even the algorithm is invalid.

It can be seen from Figure 7 that the estimated values are in good agreement with the actual values, and the estimated disturbance is nearly within the 0.5-degree range of the real values, which shows that the proposed method can correctly estimate the elevation angle and azimuth angles of the incident signals. 


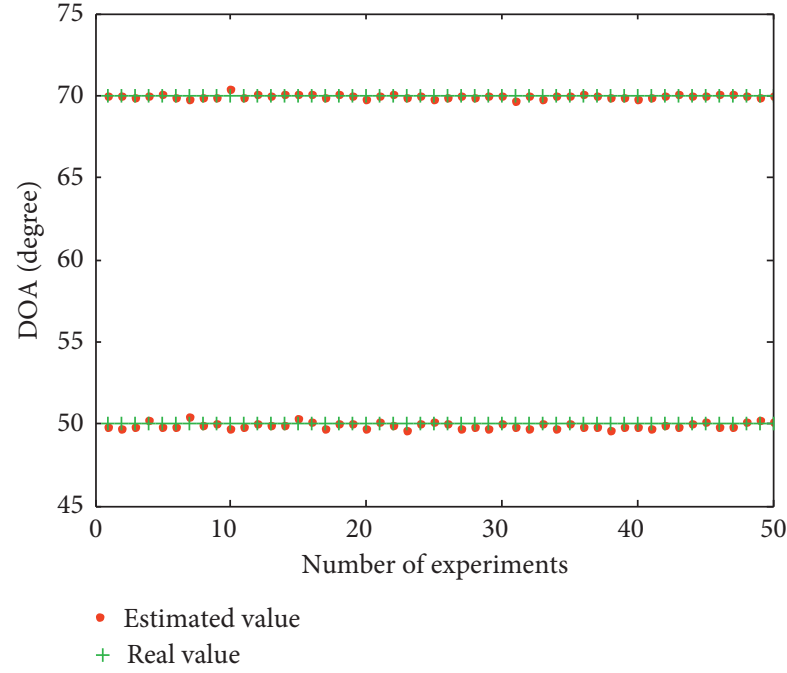

(a)

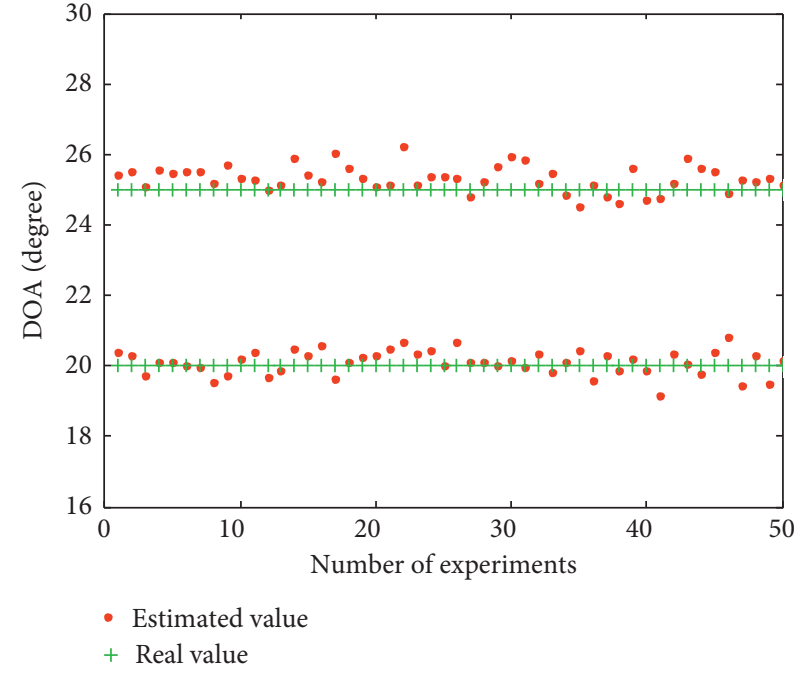

(b)

FIGURE 7: The DOA estimation versus the number of experiments.

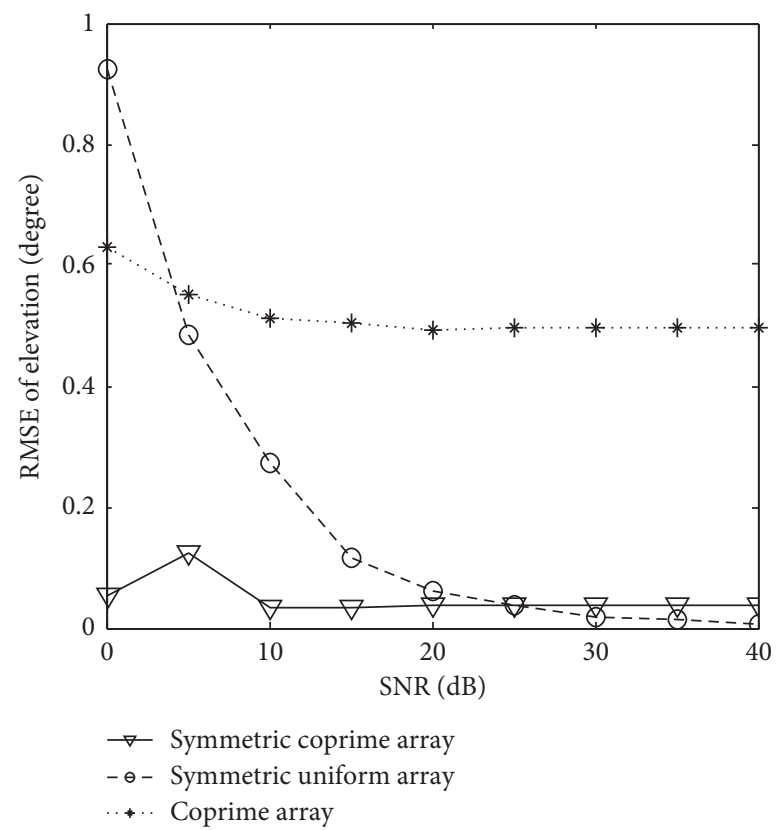

Figure 8: The RMSE of elevation versus SNR.

Simulation 2. The variation of RMSE of elevation and azimuth with SNR is studied. The snapshot number is set as 200 , and the SNR varies from $0 \mathrm{~dB}$ to $40 \mathrm{~dB}$. The array element position of the symmetric coprime array is $(0,2 d, 3 d$, $4 d)$. By contrast, the array element position of the symmetric uniform array is set as $(0, d, 2 d, 3 \mathrm{~d})$, the received data of negative half-axis $(-d,-2 d,-3 d)$ is also obtained by virtual expansion based on the symmetry of circular signal, and virtual expansion is carried out by vectorization of the data correlation matrix. Furthermore, the array element position of the coprime array is set as $(0,2 d, 3 d, 4 d)$, and it does not make use of circular signal for virtual expansion or vectorization of the data correlation matrix for virtual expansion. The following is a comparison of the corresponding estimation methods of these three arrays.

Figures 8 and 9 are the performance analysis of the DOA estimation, estimated by the symmetric uniform array method, coprime array method, and the proposed method. The RMSE of the three array methods is compared with the change of the SNR. It can be seen that the parameter estimation performance of the symmetric coprime array method and the symmetric uniform array method has been 


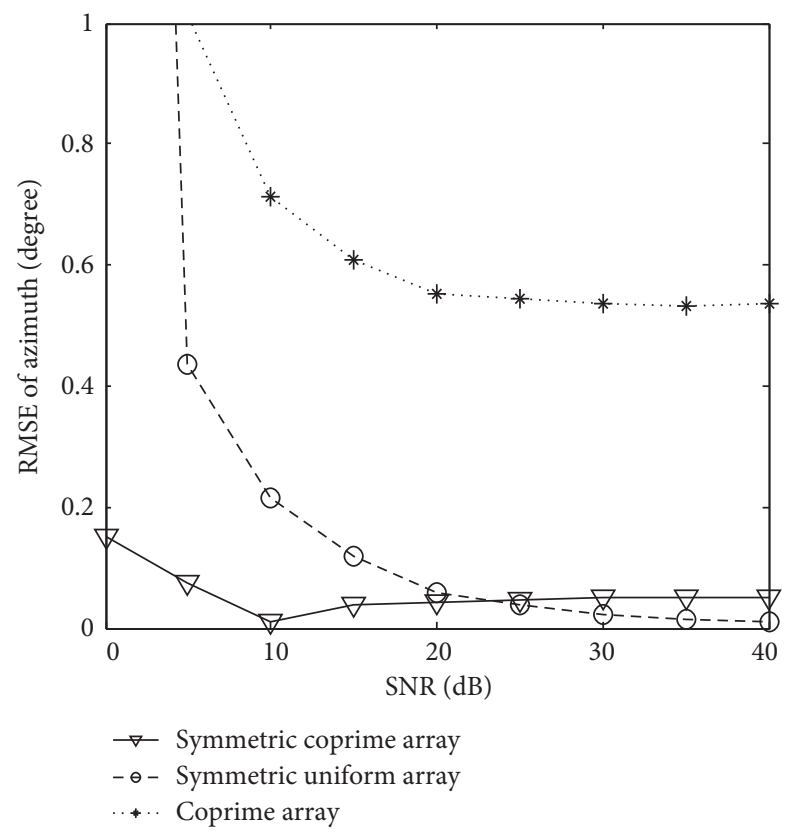

FIgURE 9: The RMSE of azimuth versus SNR.

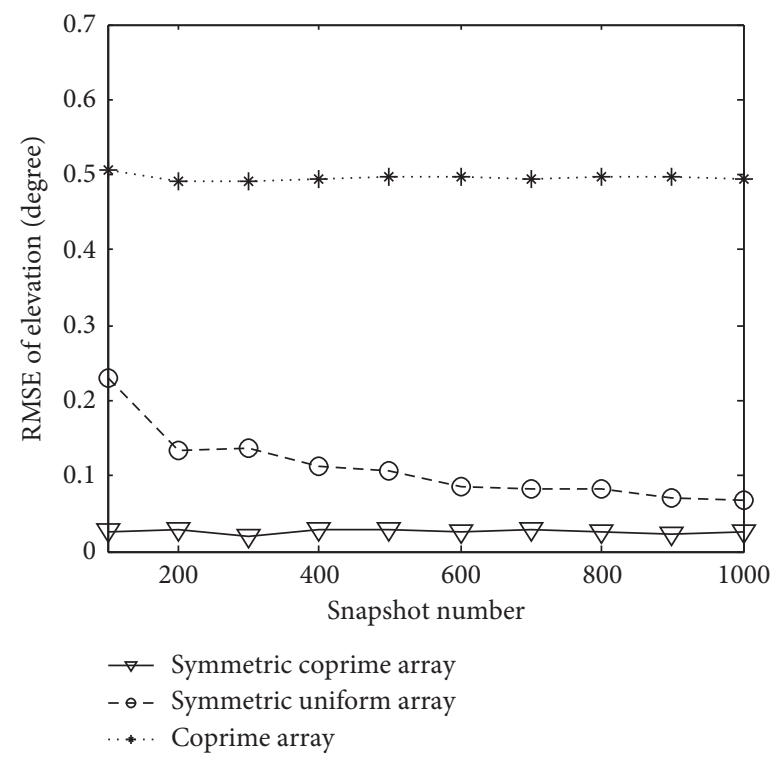

Figure 10: The RMSE of elevation versus snapshot number.

improved significantly; especially, in the low SNR, the estimation accuracy of the symmetric coprime array method is significantly higher than that of the method of the symmetric uniform array and coprime array. The RMSE of DOA estimation of the coprime array does not decrease with the increase of SNR, which is biased estimation.

The simulation results also show that there is little difference in the calculation time of the three methods. The average time of the symmetric uniform array is $2.346 \mathrm{~s}$, coprime array is $2.458 \mathrm{~s}$, and symmetric coprime array is
$2.509 \mathrm{~s}$. DOA estimation-time increase of the coprime and symmetric coprime array is mainly caused by vectorization and deredundancy.

Simulation 3. The variation of RMSE of DOA with the number of snapshots is studied, the SNR is set as $10 \mathrm{~dB}$, and the range of the snapshots is from 100 to 1000 . It can be seen from Figures 10 and 11 that when the number of snapshots reaches 200, the estimated performance almost no longer changes with the increase of the number of snapshots. The estimation error of the coprime array does not decrease with 


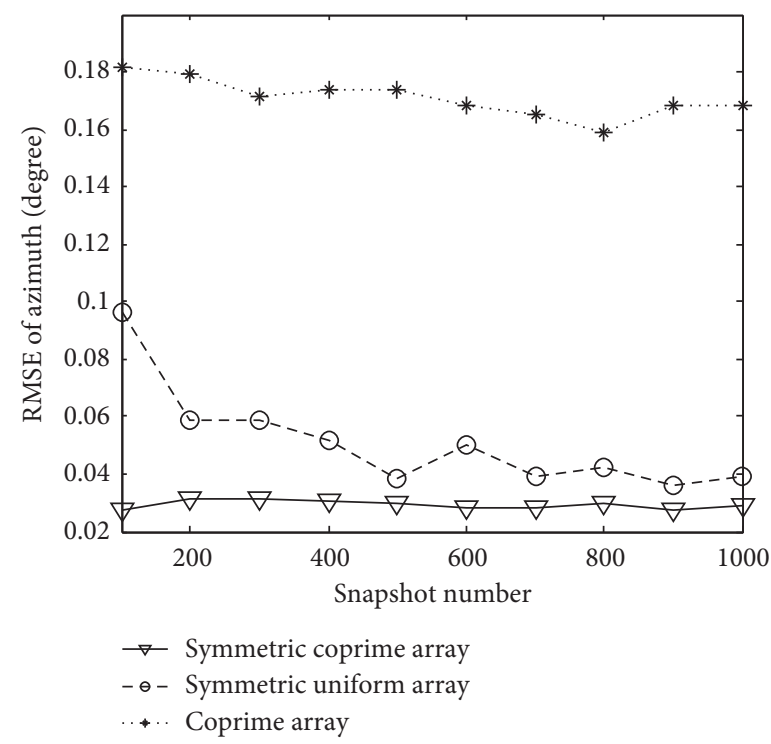

Figure 11: The RMSE of azimuth versus snapshot number.

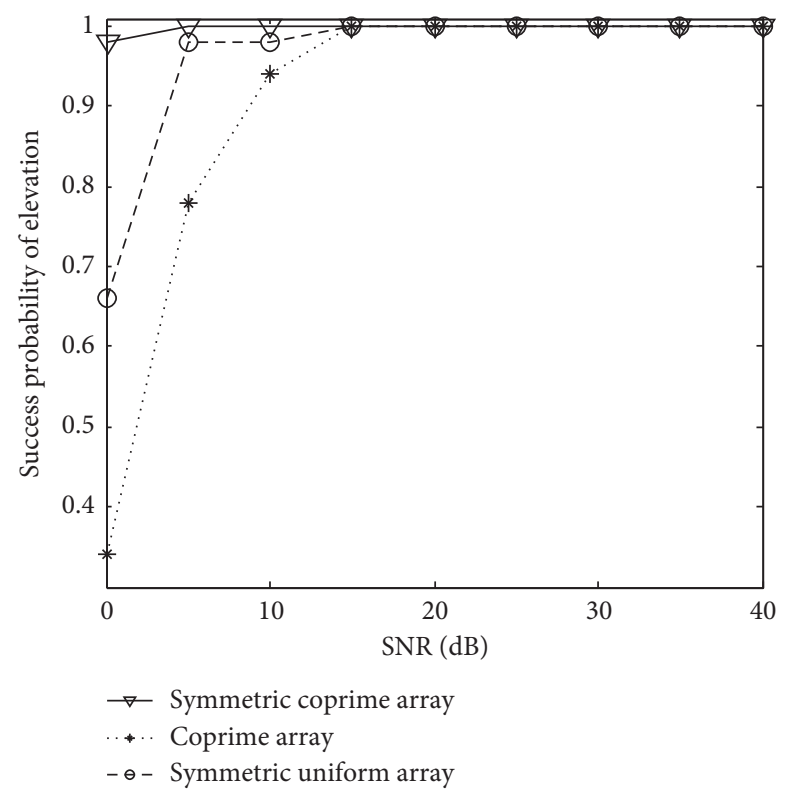

Figure 12: The probability of success of elevation.

the increase of the number of snapshots, which is biased estimation.

Simulation 4. The variation of success probability of DOA (elevation and azimuth angles) with SNR is studied, the snapshot number is set as 200 , and the SNR varies from $0 \mathrm{~dB}$ to $40 \mathrm{~dB}$.
From Figures 12 and 13, it can be seen that the DOA estimation performance of the proposed symmetric coprime array method is better than that of the other two array methods. This is because the coprime symmetric array adopted in this paper effectively expands the array aperture and increases the resolution under the same number of array elements. 


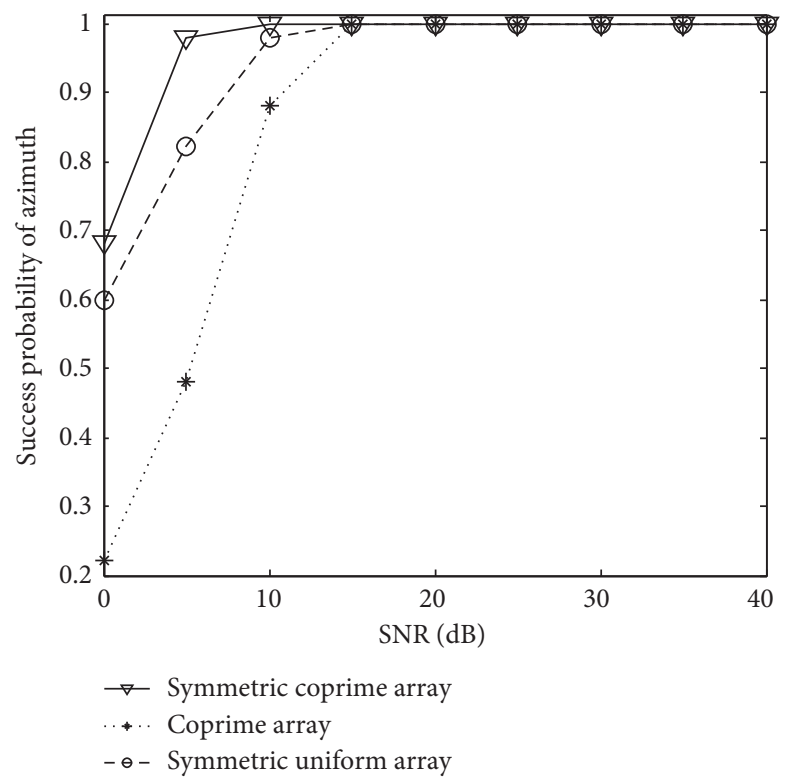

FIGURE 13: The probability of success of azimuth.

TABLE 1: The explanation of variables and notations.

\begin{tabular}{|c|c|c|c|}
\hline Variable & Meaning & Variable & Meaning \\
\hline$Z_{a}$ & The coprime subarray distributed in the $z$-axis & $Z_{a}^{\prime}$ & The virtual subarrays distributed in the $z$-axis \\
\hline & The coprime subarray distributed in the $y$-axis & $Y_{a}^{\prime}$ & The virtual subarrays distributed in the $y$-axis \\
\hline$Y_{1}(t)$ & The received signal of the $y$-axis real subarray & $Z_{1}(t)$ & The received signal of the $z$-axis real subarray \\
\hline$Y_{2}(t)$ & The received signal of the $y$-axis virtual subarray & $Z_{2}(t)$ & The received signal of the $z$-axis virtual subarray \\
\hline$Y(t)$ & The whole array received signal of the $y$-axis subarray & $Z(t)$ & The whole array received signal of the $y$-axis subarray \\
\hline$A_{y}$ & The $y$-axis real subarray steering vector & $A_{z}$ & The $z$-axis real subarray steering vector \\
\hline & The whole array steering vector of the $y$-axis subarray & $A_{z}$ & The whole array steering vector of the $z$-axis subarray \\
\hline$N_{y}(t)$ & The real $y$-axis subarray noise & $N_{z}(t)$ & The noise of the $z$-axis subarray \\
\hline$\tilde{N}_{y}^{\prime}(t)$ & The whole array noises of the $y$-axis subarray & $\tilde{N}_{z}(t)$ & The whole array noises of the $z$-axis subarray \\
\hline$R_{y y}$ & $\begin{array}{c}\text { The covariance matrix of the whole received data of the } y \text { - } \\
\text { axis subarray }\end{array}$ & $R_{z z}$ & $\begin{array}{l}\text { The covariance matrix of the whole received data of the } y \text { - } \\
\text { axis subarray }\end{array}$ \\
\hline$Y_{v}$ & $\begin{array}{c}\text { The vectorized data covariance matrix of the } y \text {-axis } \\
\text { subarray }\end{array}$ & $Z_{v}$ & $\begin{array}{l}\text { The vectorized data covariance matrix of the } y \text {-axis } \\
\text { subarray }\end{array}$ \\
\hline$\tilde{Y}_{v}$ & $\begin{array}{l}\text { The equivalent received data under the } z \text {-axis augmented } \\
\text { array }\end{array}$ & $\widetilde{Z}_{v}$ & $\begin{array}{l}\text { The equivalent received data under the } z \text {-axis augmented } \\
\text { array }\end{array}$ \\
\hline$B_{y}$ & $\begin{array}{c}\text { The Kronecker product operation of two } y \text {-axis steering } \\
\text { vectors }\end{array}$ & $B_{z}$ & $\begin{array}{c}\text { The Kronecker product operation of two } z \text {-axis steering } \\
\text { vectors }\end{array}$ \\
\hline$\widetilde{B}_{y}$ & $\begin{array}{c}\text { The } y \text {-axis steering vectors of the uniform augmented } \\
\text { array }\end{array}$ & $\widetilde{B}_{z}$ & $\begin{array}{l}\text { The } z \text {-axis steering vectors of the uniform augmented } \\
\text { array }\end{array}$ \\
\hline$\widetilde{B}_{y}$ & The overcomplete azimuth angle sparse dictionary & $\widetilde{B}_{z}$ & The overcomplete elevation angle sparse dictionary \\
\hline$y^{\prime}$ & The variance of the potential azimu & $x^{\prime}$ & The variance of the potential azimuth angle signals \\
\hline$\widehat{x}$ & The estimated elevation angle & $\widehat{y}$ & The estimated azimuth angle \\
\hline
\end{tabular}

\section{Conclusions}

In this paper, a two-dimensional DOA estimation method of the coprime array is studied. An L-shaped coprime array arranged in the $z$-axis and $y$-axis is used as a receiving array. The second-order statistic of the coprime array is used to construct the virtual augmented array to receive signals. Two-dimensional DOA estimation is realized by the sparse reconstruction method and optimal solution by minimum absolute convergence and selection operator. The decoupling of elevation and azimuth angles is realized by using the advantage that the $z$-axis subarray is only related to elevation angle. The two-dimensional parameter estimation is realized by two one-dimensional sparse reconstructions, and the parameters are automatically paired, and no additional pairing operation is needed. It can be seen that if the coprime array does not use the vectorization of the covariance matrix for the virtual expansion of the array, there will be ambiguity in the DOA estimation, resulting in the biased estimation. However, the proposed method greatly improves the freedom of the array on the premise of maintaining the same number of elements as that of the two other array methods. The proposed method also improves the accuracy and resolution of DOA estimation under low SNR. 


\section{Appendix}

Due to the great amount of different variables and notations in this manuscript, some points are hard to be followed by the reader, so Table 1 is introduced to facilitate the reader to read.

\section{Data Availability}

All data included in this study are available upon request by contact with the corresponding author.

\section{Conflicts of Interest}

The authors declare no potential conflicts of interest with respect to the research, authorship, and/or publication of this article.

\section{Acknowledgments}

This work was supported by the National Natural Science Foundation of China under Contract nos. 61772398 and 61972239, the Key Research and Development Program Projects of Shaanxi Province (2019SF-257, 2020GY-024, and 2021GY-182), the Special Scientific Research Project of the Shaanxi Provincial Education Department (19JK0169), and the Science and Technology Program of Hantai District (2019KX-21).

\section{References}

[1] K. T. Wong, L. S. Linshan Li, and M. D. Zoltowski, "RootMUSIC-based direction-finding and polarization estimation using diversely polarized possibly collocated antennas," IEEE Antennas and Wireless Propagation Letters, vol. 3, pp. 129132, 2004.

[2] L. Wang, G. Wang, and Z. Chen, "Joint DOA-polarization estimation based on uniform concentric circular array," Journal of Electromagnetic Waves and Applications, vol. 27, no. 13, pp. 1702-1714, 2013.

[3] X. Yuan, K. T. Wong, and K. Agrawal, "Polarization estimation with a dipole-dipole pair, a dipole-loop pair, or a loop-loop pair of various orientations," IEEE Transactions on Antennas and Propagation, vol. 60, no. 5, pp. 2442-2452, 2012.

[4] K. T. Wong and A. K.-Y. Lai, "Inexpensive upgrade of basestation dumb antennas by two magnetic loops for "blind" adaptive downlink beamforming," IEEE Antennas and Propagation Magazine, vol. 47, no. 1, pp. 189-193, 2005.

[5] F. G. Sun, Q. H. Wu, Y. M. Sun, G. R. Ding, and P. Lan, “An iterative approach for sparse direction-of- arrival estimation in coprime arrays with off-grid targets," Digital Signal Processing, vol. 61, pp. 35-42, 2016.

[6] C.-L. Liu and P. P. Vaidyanathan, "Cramér-Rao bounds for coprime and other sparse arrays, which find more sources than sensors," Digital Signal Processing, vol. 61, pp. 43-61, 2017.

[7] P. Pal and P. P. Vaidyanathan, "Nested arrays: a novel approach to array processing with enhanced degrees of freedom," IEEE Transactions on Signal Processing, vol. 58, no. 8, pp. 4167-4181, 2010.
[8] F. Sun, P. Lan, and B. Gao, "Partial spectral search-based DOA estimation method for co-prime linear arrays," Electronics Letters, vol. 51, no. 24, pp. 2053-2055, 2015.

[9] P. P. Vaidyanathan and P. Pal, "Sparse sensing with coprime arrays," in Proceedings of the IEEE 44th Asilomar Conference on Signals Systems and Computers, pp. 1405-1409, Pacific Grove, CA, USA, November 2010.

[10] P. Pal and P. P. Vaidyanathan, "Coprime Sampling and the Music Algorithm," in Proceedings of the 2011 Digital Signal Processing and Signal Processing Education Meeting, pp. 289-294, Sedona, AZ, USA, January 2011.

[11] H. Zhang and C. X. Wu, "DOA estimation based on coprime array of covariance matrix reconstruction," Journal of Detection and Control, vol. 40, no. 5, pp. 64-68, 2018.

[12] Q. H. Liu, X. D. Yi, L. N. Jin, and W. Chen, "Two dimensional direction of arrival estimation for co-prime L-shaped array using sparse reconstruction," in Proceedings of the IEEE 8th International Congress on Image and Signal Processing (CISP), pp. 1499-1503, Shenyang, China, October 2015.

[13] J. Liang and D. Liu, "Passive localization of near-field sources using cumulant," IEEE Sensors Journal, vol. 9, no. 8, pp. 953-960, 2009.

[14] H. Shao, W. M. Su, H. Gu, and C. Wang, "Two-dimensional direction finding using a sparse coprime L-sharped array [J]," Chinese Journal of Radio Science, vol. 27, no. 5, pp. 886-891, 2012.

[15] Y. Yue, Y. Xu, L. Shen, and Z. Liu, "Parameter estimation of coexisted circular and strictly non-circular signals," Electronics Letters, vol. 53, no. 13, pp. 864-866, 2017.

[16] X. Yang, Z. Zheng, and B. Hu, "Off-grid DOA estimation of incoherently distributed non-circular sources via generalised approximate message passing," Electronics Letters, vol. 52, no. 4, pp. 262-264, 2016.

[17] P. Sebastian, H. Martin, and M. Carlos, "DOA Estimation using random linear arrays via compressive sensing [J]," IEEE Latin America Transactions, vol. 12, no. 5, pp. 859-863, 2014.

[18] E. T. Northardt, I. Bilik, and Y. I. Abramovich, "Spatial compressive sensing for direction-of-arrival estimation with bias mitigation via expected likelihood," IEEE Transactions on Signal Processing, vol. 61, no. 5, pp. 1183-1195, 2013. 